\title{
Angiomiolipoma renal con invasión de vena renal y vena cava inferior. Presentación de un caso y revisión de la literatura
}

\section{Renal Angiomyolipoma with Extension to Renal and Cava Veins. A Case Report and Revision of the Literature}

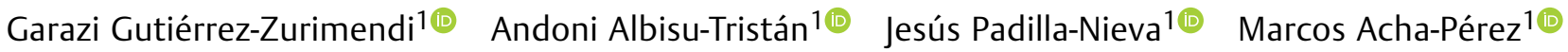 \\ Aranzazu Urresola-Olabarrieta ${ }^{2}$ () Gonzalo García de Casasola-Rodríguez ${ }^{3(0)}$ Antonio Arruza-Echevarría ${ }^{1 \odot}$
}

${ }^{1}$ Servicio de Urología, Hospital Universitario Cruces, Barakaldo, País Vasco, España

2 Servicio de Radiodiagnóstico, Hospital Universitario Cruces, Barakaldo, País Vasco, España

${ }^{3}$ Servicio de Anatomía Patológica, Hospital Universitario Cruces,

Barakaldo, País Vasco, España
Address for correspondence Garazi Gutiérrez-Zurimendi, Servicio de Urología, Hospital Universitario Cruces-Gurutzeta, Plaza de Cruces, S/N, 48903 Barakaldo, Bizkaia, España (e-mail: garazigz@gmail.com).

Urol Colomb 2020;29:99-102.

\section{Resumen}

\section{Palabras Clave}

- angiomiolipoma

- trombo graso

- invasión venosa

- trombectomía

- cavotomía

\section{Abstract}

Introducción El angiomiolipoma es un tumor benigno cuya presentación con invasión del seno renal y formación de trombo venoso tumoral es muy infrecuente, habiéndose descrito aproximadamente 50 casos desde que en 1982 Kutcher describiera el primero.

Reporte de caso Presentamos un caso angiomiolipoma renal con afectación venosa diagnosticado mediante TAC a partir del hallazgo de trombo venoso en ecografía y resonancia en una paciente de 43 años embarazada y asintomática que presentó elevación de transminasas en una analítica. Ante la posibilidad de que se tratara de una variante epitelioide y/o clásica con potencial de malignización, se decidió tratamiento mediante nefrectomía y trombectomía abierta, sin incidencias intra ni postoperatorias. La paciente se encuentra asintomática tras 6 meses de seguimiento. La histología fue compatible con angiomiolipoma clásico con trombo venoso.

Conclusión La invasión de vena renal por un angiomiolipoma es extremadamente rara. Aunque infrecuente, habría que considerar la posibilidad de malignización para no demorar su tratamiento.

Introduction Angiomyolipoma is a benign kidney tumour that rarely affects the renal sinus or the renal or caval vein. Kutcher made the first case report in 1982, and since then there have been described approximately 50 cases.

Case Report We present a case of angiomyolipoma with venous thrombus in a 43 years-old asymptomatic pregnant woman who presented elevation of transaminases in

\section{received}

October 6, 2019

accepted

November 19, 2019
DOI https://doi.org/

$10.1055 / \mathrm{s}-0040-1701277$.

ISSN 0120-789X.

e ISSN 2027-0119.
Copyright ( 2020 , Sociedad Colombiana License terms de Urología. Publicado por Thieme Revinter Publicações Ltda., Rio de Janeiro, Brazil. Todos los derechos reservados.

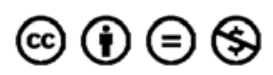


Keywords

- angiomyolipoma

- fat thrombus

- venous invasion

- thrombectomy

- cavotomy a blood test, with an ultrasonography that showed a venous thrombus without any renal mass, being diagnosed after giving birth by scanner. Having in account the possibility of malignancy of the mass, we performed surgical treatment by open radical nephrectomy and thrombectomy, without intraoperative nor postoperative incidences. The patient remains asymptomatic 6 months after surgery. The histology was compatible with classic angiomyolipoma with venous thrombus.

Conclussion Angiomyolipoma causing venous invasion is extremely rare, but we should take into consideration its possibility of malignancy in order to treat it as soon as possible.

\section{Introducción}

El angiomiolipoma (AML) es un tumor benigno del mesénquima renal formado por tejido adiposo, vascular y músculo liso. ${ }^{1-3}$ Es poco frecuente, generalmente asintomático aunque potencialmente peligroso ya que su mayor complicación es el sangrado retroperitoneal por rotura espontánea cuando su tamaño supera los $4 \mathrm{~cm}$. Aunque se consideran de naturaleza benigna y habitualmente se localizan en el parénquima renal, se han descrito casos de AML clásicos localmente invasivos con afectación de seno renal, grasa perirrenal e incluso vena renal con posibilidad de extensión a cava inferior, habiéndose descrito aproximadamente 50 casos desde que en 1982 Kutcher describiera el primero. La variante epitelioide es histológicamente más agresiva, con mayor riesgo potencial de malignización y metástasis a distancia. ${ }^{3,4}$

El tratamiento está indicado en lesiones mayores de $4 \mathrm{~cm}$ con riesgo de rotura, en casos sintomáticos o cuando el diagnóstico es dudoso o se sospecha malignidad. Este se puede realizar mediante embolización selectiva preventiva cuando se prevé riesgo de rotura o de manera urgente ante rotura espontánea del mismo, así como mediante nefrectomía parcial o radical. En casos de AML con trombo tumoral en vena renal y/o VCI está indicado realizar nefrectomía y trombectomía del trombo tumoral. ${ }^{3}$

Presentamos un caso angiomiolipoma renal con afectación venosa diagnosticado en una paciente de 43 años durante su embarazo.

\section{Presentación Del Caso Clínico}

Presentamos el caso de una mujer embarazada de 43 años con antecedentes de asma e intervenida de adenoidectomía en la infancia, que en el verano de 2017 se encontraba en seguimiento estrecho por obstetricia por diabetes gestacional y test de Coombs + , para prevención de enfermedad hemolítica perinatal. En la semana 38 del embarazo, a raíz de una analítica con elevación de transaminasas estando la paciente asintomática, se realiza una ecografía abdominal con hallazgo incidental de trombo en vena renal derecha hasta $\mathrm{VCI}$, por lo que ingresa para completar estudio. Se realiza una resonancia magnética (RM) en la que se describe una ocupación y distensión de la totalidad de la vena renal derecha que se extiende por la vena cava inferior suprarrenal e infrahepática, con extensión longitudinal afectando aproximadamente $6 \mathrm{~cm}$ de la vena cava, sugestivo de trombosis en dicha localización, sin verse masas exofíticas renales ni otros hallazgos.

Ante esos hallazgos, es valorada por los servicios de cirugía vascular y radiología intervencionista, quienes inician anticoagulación a dosis terapéuticas y desestiman la colocación de filtro de vena cava por no ser un procedimiento seguro debido a la extensión craneal del trombo.

Se decide programar el parto vía vaginal, que se lleva a cabo 8 días después del ingreso con ayuda de ventosa, sin incidencias.

Dos días después del parto, se realiza angioTAC para filiar origen del trombo venoso, con los siguientes hallazgos: masa de naturaleza grasa en seno renal derecho afectando al polo superior que rodea el infundíbulo de aprox. $45 \times 28 \mathrm{~mm}$ y se continúa con trombo graso que ocupa la vena renal derecha y vena cava intrahepática con extensión craneocaudal de 5$6 \mathrm{~cm}$. Se interpretan los hallazgos como un tumor renal tipo angiomiolipoma infiltrando la vena renal y la vena cava inferior, sin datos de tromboembolismo pulmonar. (-Figura 1)

La paciente es dada de alta tras el parto y se cita para control 2 meses después, realizándose angioTAC sin cambios respecto a previo, por lo que se propone a la paciente nefrectomía derecha y trombectomía.

Se realiza la cirugía mediante laparotomía con acceso tipo chevrón, realizando nefrectomía radical derecha y trombectomía con extracción de trombo de aspecto graso mediante cavotomía, sin incidencias intraoperatorias. El tiempo operatorio fue de 210 minutos y el sangrado estimado de 500cc. La paciente presentó buena evolución postoperatoria, siendo dada de alta 11 días después de la cirugía, sin necesidad de reingreso y con buena evolución en el control postoperatorio. (- Figura 2)

El resultado anatomopatológico fue compatible con angiomiolipoma clásico de predominio adiposo, con invasión de vena renal y vena cava, siendo el trombo analizado compatible con angiomiolipoma. (-Figura 3) 

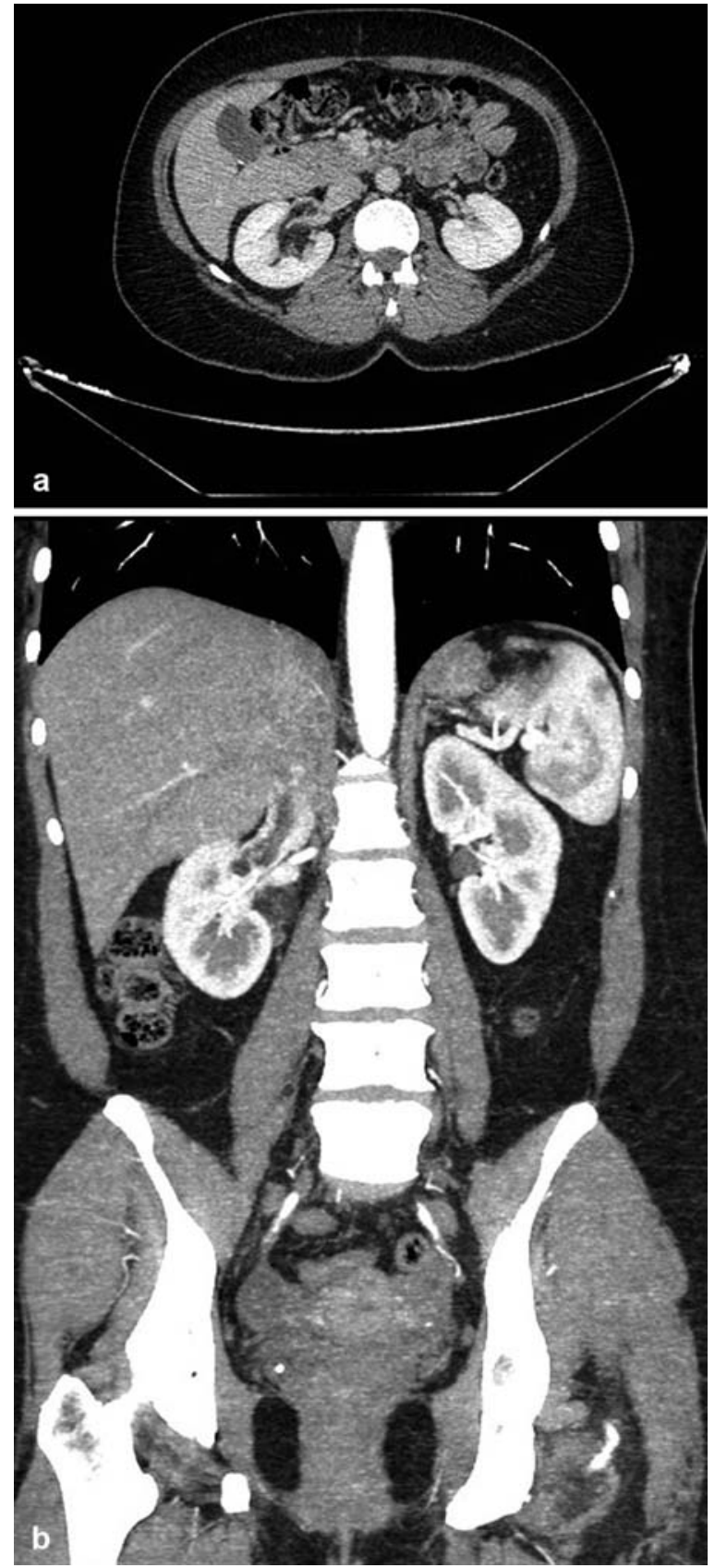

Fig. 1 (a y b) Cortes axial y coronal de TAC con contraste iodado i.v.: masa de naturaleza grasa en seno renal derecho con extensión intravascular a vena renal derecha y vena cava inferior visualizando ocupación de la luz vascular por tejido de densidad grasa.

\section{Discusión}

El angiomiolipoma (AML) es un tumor benigno del mesénquima renal formado por tejido adiposo, vascular $\mathrm{y}$ músculo liso, considerado un hamartoma constituido por proliferación de células epitelioides perivasculares ("PEComa"), que puede tener un tamaño variable entre $2 \mathrm{~mm}$ a $20 \mathrm{~cm}$. Fue descrito por primera vez por Morgan en $19510^{3,4}$

Se trata de una entidad infrecuente (0,3-3\% de los tumores renales), que normalmente cursa de manera asintomática, por lo que su diagnóstico habitual suele ser incidental. ${ }^{3,4} \mathrm{Sin}$ embargo, en ocasiones se diagnostica de urgencia ya que es la

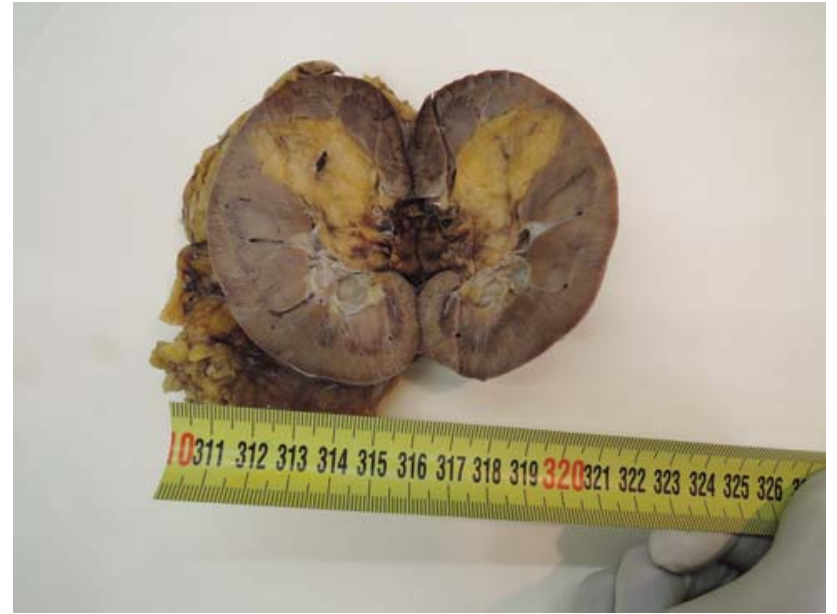

Fig. 2 Imagen macroscópica de riñón derecho tras nefrectomía, con hallazgo de angiomiolipoma de predominio adiposo en seno renal con invasión de vena renal.

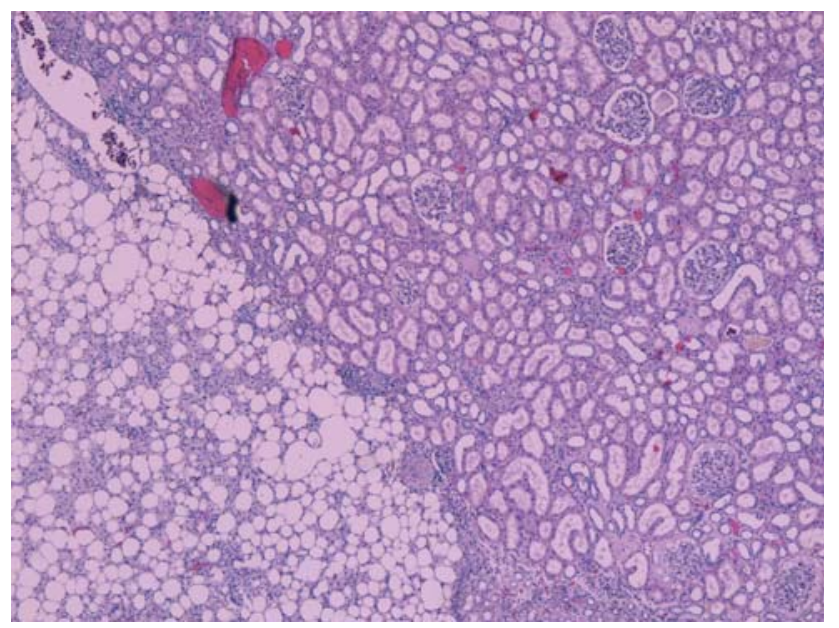

Fig. 3 Imagen de microscopía óptica con hallazgo de estructuras compatibles con angiomiolipoma renal clásico de predominio adiposo.

causa más frecuente de rotura renal espontánea cuando superan $\operatorname{los} 4 \mathrm{~cm}^{5}$

Su localización más frecuente es el parénquima renal, siendo raro en el seno renal, por lo que en esos casos debe realizarse diagnóstico diferencial con lipomatosis, lipoma y liposarcoma, que pueden presentar características similares. 3,4

Existen dos formas de presentación del angiomiolipoma: la forma esporádica y la relacionada con la Esclerosis Tuberosa (ET). ${ }^{1-5}$ La presentación más habitual es la esporádica, que aparece con mayor frecuencia en mujeres (relación 4:1), entre los 45-55 años y normalmente siendo lesiones de mayor tamaño y con mayor tendencia a la hemorragia. Se ha relacionado la existencia de angiomiolipoma con la influencia de hormonas femeninas, habiéndose observado un incremento del crecimiento y posibilidad de rotura durante el embarazo o tras tratamiento con estrógenos, así como asociado a la linfangioleiomiomatosis (también relacionada con las hormonas femeninas), recomendándose controles periódicos para evitar sus complicaciones. ${ }^{3,4}$ 
Los angiomiolipomas relacionados con el síndrome de Esclerosis Tuberosa se manifiestan en igual porcentaje en ambos sexos y a una edad más temprana (25-35 años), siendo habitualmente más pequeños, multifocales y, en ocasiones, bilaterales. $^{1,3}$

Su sospecha diagnóstica se basa en pruebas de imagen, aunque su diagnóstico definitivo es anatomopatológico, debiendo realizar un diagnóstico diferencial con el cáncer renal de células claras, oncocitoma, metástasis de tumor primario. ${ }^{6}$

Según su histología, el angiomiolipoma se ha clasificado como clásico o epitelioide. El primero suele tener un comportamiento benigno, con un crecimiento lento y desarrollo local no invasivo, aunque en contadas ocasiones puede ser localmente invasivo con extensión a grasa perirrenal, sistema colector, vena renal hacia cava inferior (VCI) y/o aurícula derecha e incluso afectar a ganglios y/o bazo. El AML epitelioide (AMLE) es menos frecuente pero mucho más agresivo, y se asocia en más de la mitad de los casos con la Esclerosis Tuberosa. Tiene mayor potencial de malignización, pudiendo convertirse en angiomioliposarcoma y/o causar invasión local, afectación de ganglios regionales o incluso metástasis a distancia. Por ese motivo, precisa un seguimiento estrecho tras su tratamiento por el riesgo de recurrencia local o sistémica. ${ }^{5,6}$

El tratamiento se puede realizar mediante embolización selectiva preventiva cuando se prevé riesgo de rotura o de manera urgente ante rotura espontánea del mismo, así como mediante nefrectomía parcial o radical. ${ }^{3}$

En casos de AML con trombo tumoral en vena renal y/o VCI está indicado realizar nefrectomía y trombectomía, generalmente mediante laparotomía, aunque se han descrito casos mediante laparoscopia con los mismos resultados oncológicos. ${ }^{4}$ En los casos en que exista trombo en cava, se podría valorar la colocación de un filtro de $\mathrm{VCI}$, siendo determinante el nivel craneal que alcanza el trombo y la afectación de la pared vascular. 3,4

Asimismo, en el caso de los AML irresecables y/o malignos, Ikarashi y col., demostraron la efectividad de los inhibidores de mTOR (everolimus, sirolimus...) como tratamiento coadyuvante para reducir la masa y convertirla en abordable - resecable quirúrgicamente. ${ }^{7}$

Describimos un caso raro de tumor renal diagnosticado mediante TAC a partir del hallazgo de trombo venoso con ecografía y resonancia, sin verse en esas pruebas la masa renal origen del trombo. El manejo se realizó mediante nefrectomía y trombectomía abierta, con anatomía patológica compatible con angiomiolipoma clásico con trombo venoso graso. Ese tipo de AML con invasión de seno renal y formación de trombo venoso tumoral es muy infrecuente, hallando en la literatura aproximadamente 50 casos descritos desde que en 1982 Kutcher describiera el primer caso. ${ }^{3,4}$

\section{Conclusiones}

El angiomiolipoma con invasión de vena renal es extremadamente infrecuente. Ante la sospecha de angiomiolipoma localmente invasivo con afectación vascular, hay que tener en cuenta la posibilidad de que se trate de una variante epitelioide y su potencial de malignización. En esos casos estaría indicada la nefrectomía y trombectomía, realizándose habitualmente vía abierta, aunque se han descrito casos de nefrectomía laparoscópica con similares resultados oncológicos.

Conflicto de intereses

Los autores declaran no tener ningún conflicto de intereses.

\section{Referencias}

1 Quicios Dorado C, Allona Almagro A. Angiomiolipoma renal causante de trombo en cava y síndrome de Budd-Chiari secundario. Arch Esp Urol 2008;61(03):435-439

2 Tan YS, Yip KH, Tan PH, Cheng WS. A right renal angiomyolipoma with IVC thrombus and pulmonary embolism. Int Urol Nephrol 2010;42(02):305-308

3 Celik SU, Kocaay AF, Sevim Y, Cetinkaya OA, Atman ED, Alacayir I. Renal angiomyolipoma with caval extension and pulmonary fat embolism. Medicine (Baltimore) 2015;94(31):e1078

4 Ruiz-Tagle D, Cauwelaert RRV, Sanhueza LV, Castilo COA. Nefrectomía radical laparoscópica en angiomiolipoma renal con invasión de vena renal. Rev Chil Cir Vol 2015;67(06): 635-637

5 Innocenti CF, Alarcón CE, Arias OE, et al. Angiomiolipoma renal con extensión tumoral a vena cava inferior y aurícula derecha. Rev Chil Cir 2008;60:150-153

6 Chandrasoma S, Daneshmand S, Wilson S, Skinner EC. Renal angiomyolipoma with liposarcomatous transformation: a case report and review of the literature. Urol Oncol 2004;22(05): 425-427

7 Ikarashi D, Mue Y, Shiomi E, et al. Efficacy of everolimus for treating renal angiomyolipoma with inferior vena cava thrombus associated with tuberous sclerosis: A case report. Urol Case Rep 2017;11:11-13 